\title{
Participation and Performance Trends in the ITU Duathlon World Championship From 2003 to 2017
}

\author{
Nikolaidis, Pantelis Theodoros ; Villiger, Elias ; Knechtle, Beat
}

\begin{abstract}
Nikolaidis, PT, Villiger, E, and Knechtle, B. Participation and performance trends in the ITU Duathlon World Championship from 2003 to 2017. J Strength Cond Res XX(X): 000-000, 2018-Participation and performance across years have been studied extensively in various endurance and ultra-endurance sports; however, less information exists with regards to duathlon (i.e., Run 1, Bike, and Run 2). The aim of this study was to examine performance and participation trends of duathletes competing either to short (10-km Run 1,50-km Bike, and 5-km Run 2) or to long distance (10-km Run 1, 150-km Bike, and 30-km Run 2) in the Powerman World Championship "Powerman Zofingen." We analyzed 7,951 finishers (women, $\mathrm{n}=1,236$, age $36.7 \pm 9.1$ years; men, $\mathrm{n}=6,715,40.1 \pm 10.1$ years) competing in "Powerman Zofingen" from 2003 to 2017. Men were faster than women by $8.2 \%(171 \pm 21$ minutes vs. $186 \pm 21$ minutes, $\mathrm{p}<0.001, \eta=0.068)$ and $7.5 \%(502 \pm 57$ minutes vs. $543 \pm 64$ minutes, $\mathrm{p}<0.001, \eta=0.068)$ in the short and long distances, respectively. Women were younger than men by 4.6 years $(35.0 \pm 9.0$ years vs. $39.6 \pm 10.5$ years, $p<0.001, \eta=0.026)$ and 1.8 years $(38.8 \pm 8.7$ years vs. $40.6 \pm 9.5$ years, $\mathrm{p}<0.001, \eta=0.005)$ in the short and long distances, respectively. An increase of women finishers across years in the long distance was observed (e.g., $n=19$ in 2003 and $n=58$ in 2017; $p<0.001$ ), whereas no change was shown in short distance and men finishers. The men-to-women ratio (MWR) decreased across years in the long, but not in the short distance. No change of race time across years was observed. The sex difference in race time increased in long distance $(\mathrm{p}=0.014)$, whereas it did not change in the short. Age increased across years in both sexes and distances $(p<0.001)$. The sex difference in age decreased in the long $(p=0.007)$, but not in the short distance. In summary, the number of women finishers increased and the MWR decreased in the long distance. The age of the finishers increased across years, and their performance remained unchanged. The increase of the sex difference in race time in the long distance might be attributed to the increased number of women finishers.
\end{abstract}

DOI: https://doi.org/10.1519/jsc.0000000000002851

Posted at the Zurich Open Repository and Archive, University of Zurich ZORA URL: https://doi.org/10.5167/uzh-157658

Journal Article

Published Version

Originally published at:

Nikolaidis, Pantelis Theodoros; Villiger, Elias; Knechtle, Beat (2021). Participation and Performance Trends in the ITU Duathlon World Championship From 2003 to 2017. Journal of Strength and Conditioning Research, 35(4):1127-1133.

DOI: https://doi.org/10.1519/jsc.0000000000002851 


\title{
Participation and Performance Trends in the ITU Duathlon World Championship From 2003 TO 2017
}

\author{
Pantelis T. Nikolaidis, ${ }^{1,2}$ Elias Villiger, ${ }^{3}$ and Beat Knechtle ${ }^{3,4}$ \\ ${ }^{1}$ Exercise Physiology Laboratory, Nikaia, Greece; ${ }^{2}$ Exercise Testing Laboratory, Hellenic Air Force Academy, Acharnes, Greece; \\ ${ }^{3}$ Institute of Primary Care, University of Zurich, Zurich, Switzerland; and ${ }^{4}$ Medbase St. Gallen am Vadianplatz, St. Gallen, \\ Switzerland
}

\begin{abstract}
Nikolaidis, PT, Villiger, E, and Knechtle, B. Participation and performance trends in the ITU Duathlon World Championship from 2003 to 2017. J Strength Cond Res XX(X): 000000, 2018-Participation and performance across years have been studied extensively in various endurance and ultra-endurance sports; however, less information exists with regards to duathlon (i.e., Run 1, Bike, and Run 2). The aim of this study was to examine performance and participation trends of duathletes competing either to short (10-km Run 1, 50-km Bike, and 5-km Run 2) or to long distance (10-km Run 1, 150-km Bike, and 30-km Run 2) in the Powerman World Championship "Powerman Zofingen." We analyzed 7,951 finishers (women, $n=1,236$, age $36.7 \pm 9.1$ years; men, $n=6,715,40.1 \pm 10.1$ years) competing in "Powerman Zofingen" from 2003 to 2017. Men were faster than women by $8.2 \%(171 \pm 21$ minutes vs. $186 \pm 21$ minutes, $\left.p<0.001, \eta^{2}=0.068\right)$ and $7.5 \%(502 \pm 57$ minutes vs. $543 \pm 64$ minutes, $\left.p<0.001, \eta^{2}=0.068\right)$ in the short and long distances, respectively. Women were younger than men by 4.6 years $(35.0 \pm 9.0$ years vs. $39.6 \pm 10.5$ years, $\left.p<0.001, \eta^{2}=0.026\right)$ and 1.8 years $(38.8 \pm 8.7$ years vs. $40.6 \pm 9.5$ years, $p<0.001, \eta^{2}=0.005$ ) in the short and long distances, respectively. An increase of women finishers across years in the long distance was observed (e.g., $n=19$ in 2003 and $n=58$ in 2017; $p<0.001$ ), whereas no change was shown in short distance and men finishers. The men-to-women ratio (MWR) decreased across years in the long, but not in the short distance. No change of race time across years was observed. The sex difference in race time increased in long distance $(p=0.014)$, whereas it did not change in the short. Age increased across years in both
\end{abstract}

Address correspondence to Dr. Beat Knechtle, beat.knechtle@hispeed.ch. $00(00) / 1-7$

Journal of Strength and Conditioning Research

(c) 2018 National Strength and Conditioning Association sexes and distances $(p<0.001)$. The sex difference in age decreased in the long $(p=0.007)$, but not in the short distance. In summary, the number of women finishers increased and the MWR decreased in the long distance. The age of the finishers increased across years, and their performance remained unchanged. The increase of the sex difference in race time in the long distance might be attributed to the increased number of women finishers.

KEY WoRDS cycling, running, sex difference, ultra-endurance

\section{INTRODUCTION}

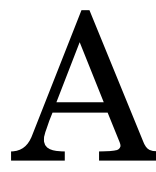

$\mathrm{n}$ increased scientific interest for duathlon, an endurance sport consisting by run-bike-run that are performed directly behind each other, has been observed during the past decades. Particularly, several aspects of this sport such as physiology $(1,3)$, biomechanics (2,6), psychology (17), sociology (8), and nutrition $(10,37)$ have been well studied, providing practical information for coaches and fitness trainers working with duathletes.

On the other hand, limited information exists about trends in participation and performance in elite duathletes competing across calendar years at top level (26). The only available study on trends in participation and performance in duathlon across calendar years has been conducted on the long-distance race of "Powerman $\mathrm{Zo}$ fingen" (10-km run, $150-\mathrm{km}$ bike, and $30-\mathrm{km}$ run) from 2002 to 2011 (26). That study of the official Powerman World Championship showed that performance remained stable during the period under examination and that men were faster than women by $\sim 17 \%$ when the annual top 10 duathletes were considered. It should be highlighted that, in addition to its long version, "Powerman Zofingen" also has a short distance consisting of 10$\mathrm{km}$ run, $50-\mathrm{km}$ bike, and $5-\mathrm{km}$ run.

Despite the abovementioned research providing some insight into the trends in participation and performance across calendar years in duathlon races, a certain gap in 
TABle 1. Participation, performance, and age of finishers by sex and distance.

\begin{tabular}{cccccc}
\hline & \multicolumn{2}{c}{ Short distance } & & \multicolumn{2}{c}{ Long distance } \\
\cline { 2 - 3 } \cline { 5 - 6 } & Women & Men & & Women & Men \\
\hline $\begin{array}{c}\text { Finishers } \\
(n)\end{array}$ & 660 & 3,490 & & 576 & 3,224 \\
Race time & $186 \pm$ & $171 \pm$ & $543 \pm$ & $502 \pm$ \\
$($ min) & 21 & 21 & 64 & 57 \\
Age (y) & $35.0 \pm$ & $39.6 \pm$ & & $38.8 \pm$ & $40.6 \pm$ \\
& 9.0 & 10.5 & & 8.7 & 9.5 \\
\hline
\end{tabular}

Values are presented as mean \pm standard deviation

the existing literature has been identified, as no information exists about the trends concerning the short distance of Powerman duathlon. Knowledge about these trends would be of practical importance for coaches and fitness trainers working with duathletes in the context of setting long-term goals.

Therefore, the aim of this study was to examine changes in participation, performance, and age of finishers in short and long version of "Powerman Zofingen" across calendar years (2003-2017). It has been previously suggested that the participation of women in another multisport (i.e., triathlon) was lower in the longer distance (18). Based on this observation, we hypothesized that women would finish in the long distance in lower rates compared with the short distance.

\section{Methods}

\section{Experimental Approach to the Problem}

To test our hypothesis, all successful female and male finishers competing in both the short- and the longdistance race in "Powerman Zofingen" were included. The "Powerman Zofingen" is a duathlon event held in Zofingen (Switzerland) within the "Powerman World Series." The long-distance race of "Powerman Zofingen" is held as the official Powerman World Championship under the name of "ITU Powerman Long Distance Duathlon World Championship."

\section{Subjects}

We analyzed data from 7,951 finishers (measured mean $\pm S D$ : women, $n=1,236$, age $36.7 \pm 9.1$ years; men, $n=$ $6,715,40.1 \pm 10.1$ years; range, $18-75$ years old) in "Powerman Zofingen" from 2003 to 2017 (Table 1). To compete in this race, athletes have to be 18 years or older. In addition, athletes must have either been registered (open category, 300 slots, first-in, first-served) at the offcial web site (www.powerman.ch/en/registration) or after being nominated by their national federation. It should be

TABLE 2. Finishers and nonfinishers by calendar year, distance, and sex.*

\begin{tabular}{|c|c|c|c|c|c|c|c|c|c|c|c|c|c|c|c|}
\hline \multirow[b]{3}{*}{ Calendar year } & \multicolumn{6}{|c|}{ Short distance } & \multicolumn{6}{|c|}{ Long distance } & \multirow{2}{*}{\multicolumn{3}{|c|}{ Total }} \\
\hline & \multicolumn{3}{|c|}{ Women } & \multicolumn{3}{|c|}{ Men } & \multicolumn{3}{|c|}{ Women } & \multicolumn{3}{|c|}{ Men } & & & \\
\hline & $\mathrm{F}(n)$ & $\mathrm{NF}(n)$ & $\%$ & $\mathrm{~F}(n)$ & $\operatorname{NF}(n)$ & $\%$ & $\mathrm{~F}(n)$ & $\mathrm{NF}(n)$ & $\%$ & $\mathrm{~F}(n)$ & $\operatorname{NF}(n)$ & $\%$ & $\mathrm{~F}(n)$ & $\mathrm{NF}(n)$ & $\%$ \\
\hline 2003 & 59 & 66 & 89.4 & 345 & 359 & 96.1 & 19 & 23 & 82.6 & 246 & 277 & 88.8 & 669 & 725 & 92.3 \\
\hline 2004 & 51 & 53 & 96.2 & 277 & 296 & 93.6 & 19 & 21 & 90.5 & 164 & 185 & 88.6 & 511 & 555 & 92.1 \\
\hline 2005 & 39 & 44 & 88.6 & 235 & 242 & 97.1 & 24 & 30 & 80.0 & 173 & 194 & 89.2 & 471 & 510 & 92.4 \\
\hline 2006 & 30 & 31 & 96.8 & 151 & 152 & 99.3 & 28 & 32 & 87.5 & 162 & 191 & 84.8 & 371 & 406 & 91.4 \\
\hline 2007 & 31 & 31 & 100.0 & 183 & 183 & 100.0 & 22 & 24 & 91.7 & 172 & 211 & 81.5 & 408 & 449 & 90.9 \\
\hline 2008 & 38 & 41 & 92.7 & 159 & 165 & 96.4 & 28 & 29 & 96.6 & 165 & 187 & 88.2 & 390 & 422 & 92.4 \\
\hline 2009 & 38 & 38 & 100.0 & 206 & 215 & 95.8 & 40 & 44 & 90.9 & 227 & 255 & 89.0 & 511 & 552 & 92.6 \\
\hline 2010 & 36 & 36 & 100.0 & 222 & 225 & 98.7 & 29 & 32 & 90.6 & 211 & 242 & 87.2 & 498 & 535 & 93.1 \\
\hline 2011 & 48 & 49 & 98.0 & 264 & 271 & 97.4 & 47 & 55 & 85.5 & 267 & 296 & 90.2 & 626 & 671 & 93.3 \\
\hline 2012 & 55 & 56 & 98.2 & 252 & 259 & 97.3 & 47 & 52 & 90.4 & 256 & 284 & 90.1 & 610 & 651 & 93.7 \\
\hline 2013 & 47 & 49 & 95.9 & 256 & 261 & 98.1 & 52 & 61 & 85.2 & 283 & 304 & 93.1 & 638 & 675 & 94.5 \\
\hline 2014 & 39 & 39 & 100.0 & 204 & 211 & 96.7 & 59 & 64 & 92.2 & 217 & 264 & 82.2 & 519 & 578 & 89.8 \\
\hline 2015 & 49 & 49 & 100.0 & 263 & 267 & 98.5 & 48 & 53 & 90.6 & 232 & 275 & 84.4 & 592 & 644 & 91.9 \\
\hline 2016 & 47 & 47 & 100.0 & 249 & 254 & 98.0 & 56 & 66 & 84.8 & 228 & 262 & 87.0 & 580 & 629 & 92.2 \\
\hline 2017 & 53 & 55 & 96.4 & 223 & 225 & 99.1 & 58 & 66 & 87.9 & 221 & 261 & 84.7 & 555 & 607 & 91.4 \\
\hline Total & 660 & 684 & 96.5 & 3,489 & 3,585 & 97.3 & 576 & 652 & 88.3 & 3,224 & 3,688 & 87.4 & 7,949 & 8,609 & 92.3 \\
\hline
\end{tabular}

${ }^{*} \mathrm{~F}=$ finishers; $\mathrm{NF}=$ nonfinishers.

2 Journal of Strength and Conditioning Research 
TABLE 3. Weather data (temperature, precipitation, and wind) for the day of the race by calendar year.*

\begin{tabular}{lccc}
\hline Year & Daily maximum temperature $\left({ }^{\circ} \mathrm{C}\right)$ & Daily precipitation $\left(\mathrm{mm} \cdot \mathrm{d}^{-1}\right)$ & Daily average wind speed $\left(\mathrm{m} \cdot \mathrm{s}^{-1}\right)$ \\
\hline 2003 & 19.6 & 0.7 & 5.1 \\
2004 & 27.2 & 1.2 & 3.6 \\
2005 & 26.4 & 1.0 & 0.0 \\
2006 & 20.9 & 13.0 & 2.9 \\
2007 & 17.4 & 0.0 & 3.4 \\
2008 & 23.3 & 0.0 & 1.9 \\
2009 & 18.5 & 0.0 & 2.8 \\
2010 & 22.4 & 0.0 & 2.8 \\
2011 & 21.5 & 38.6 & 1.4 \\
2012 & 18.3 & 0.0 & 1.6 \\
2013 & 19.1 & 18.6 & 3.4 \\
2014 & 26.0 & 0.0 & 2.0 \\
2015 & 16.6 & 0.0 & 4.3 \\
2016 & 23.8 & 4.8 & 4.4 \\
2017 & 18.9 & 0.0 & 2.0 \\
\hline
\end{tabular}

*Weather data are from Bundesamt für Meteorologie und Klimatologie, MeteoSchweiz.

highlighted that our analysis focused on finishers and excluded nonfinishers, i.e., those who were in the participants' list, but did not finish the race for unknown reason. Rates of finishers are presented in Table 2. All procedures used for the study were approved by the institutional review board of Kanton St. Gallen, Switzerland, with a waiver from the requirement for informed consent of the finishers given the fact that the study involved the analysis of publicly available data. The study was conducted in accordance with recognized ethical standards according to the Declaration of Helsinki adopted in 1964 and revised in 2013 (Table 3).

\section{Procedures}

Data were obtained from the official race web site from "Powerman Zofingen" www.powerman.ch/de. Since 2003, the short and long versions of "Powerman Zofingen" have the same formats, whereas before that year, the race course and the distances of the split disciplines changed several times since the first edition of the race in 1989. Therefore, to have comparable data, we restricted the race results to 2003-2017. Moreover, data on weather condition (i.e., temperature, precipitation, and wind) for the day of race were provided by the Bundesamt für Meteorologie und Klimatologie, MeteoSchweiz (Table 3).

\section{Statistical Analyses}

Data are presented as mean values $\pm S D$ s. All data were checked for normality using the Kolmogorov-Smirnov test, and visual inspection of $Q-Q$ plots and, thereafter, parametric statistics were used. Chi square examined sex $\times$ calendar year association. Within each distance, a 2-way analysis of variance examined the effects of sex and calendar year on race time and age. The magnitude of these differences was

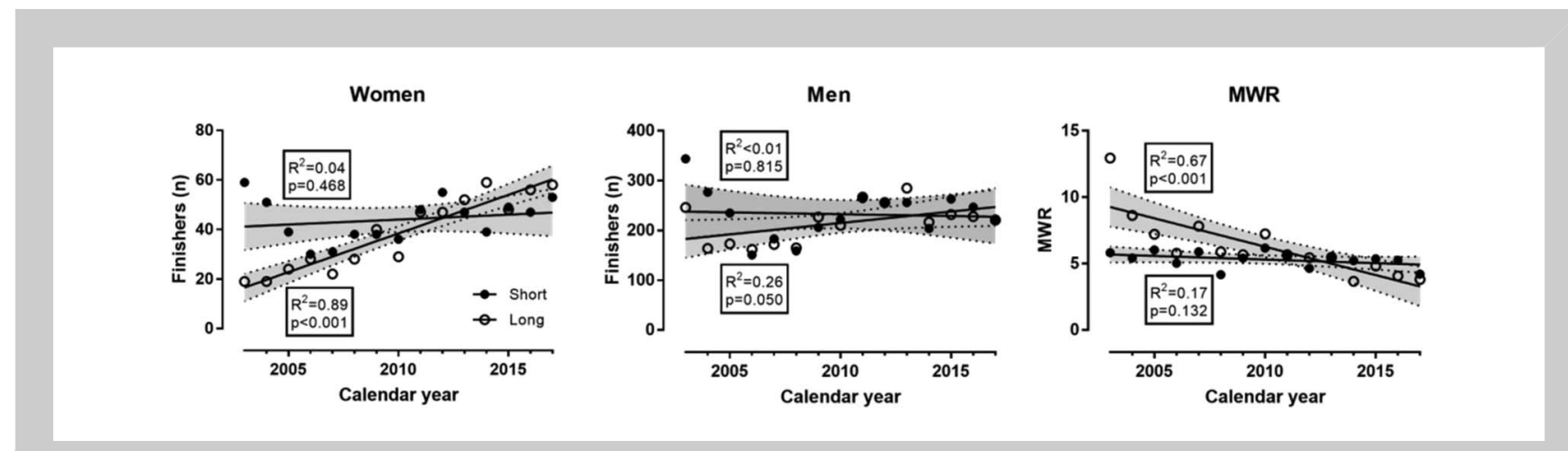

Figure 1. Finishers by calendar year and short vs. long distance in women and men. The dotted lines and gray areas represent $95 \%$ confidence intervals. $R^{2}=$ correlation of determination; MWR = men-to-women ratio. 

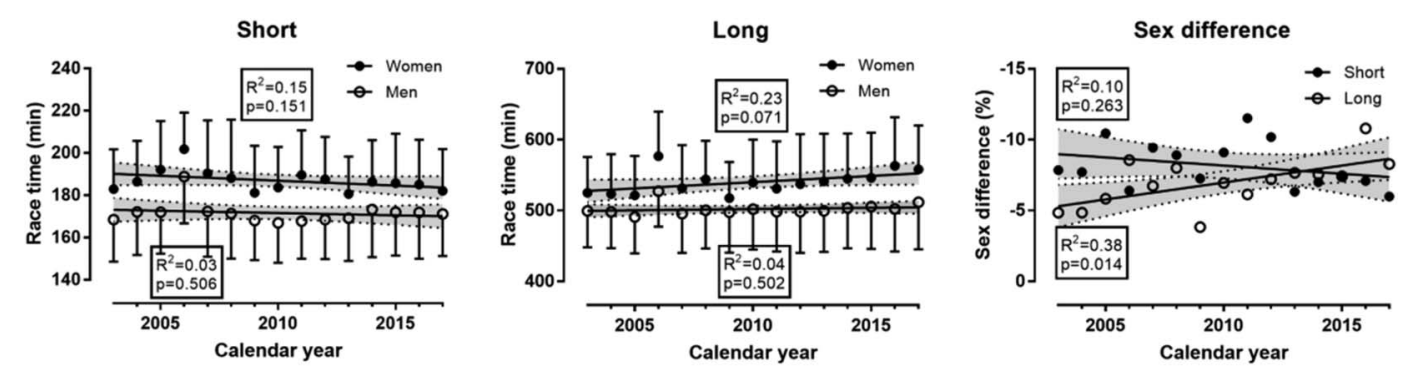

Figure 2. Race time by calendar year and short vs. long distance in women and men. The dotted lines and gray areas represent $95 \%$ confidence intervals. Error bars show $S D$ s. $R^{2}=$ correlation of determination. Sex difference in performance was calculated as "100 $\times$ (race time in men - race time in women)/race time in women."

examined using effect size partial eta square $\left(\eta_{p}^{2}\right)$ and was evaluated as following: small $\left(0.010<\eta_{p}^{2} \leq 0.059\right)$, moderate $\left(0.059<\eta_{p}^{2} \leq 0.138\right)$, and large $\left(\eta_{p}^{2}>0.138\right)(9)$. Pearson correlation coefficient $r$ examined the relationship of race time, age, and sex differences with calendar year, as well as the relationship of race time with weather conditions (temperature, precipitation, and wind). Statistical analyses were performed using GraphPad Prism v. 7.0 (GraphPad Software, San Diego, CA, USA) and IBM SPSS v.23.0 (SPSS, Chicago, IL, USA).

\section{Results}

An increase of women finishers across years in the long distance was observed (e.g., $n=19$ in 2003 and $n=58$ in 2017; $p<0.001$ ), whereas no change was shown in short distance and men finishers (Figure 1). The men-to-women ratio (MWR) decreased across years in the long, but not in the short distance. A sex $\times$ calendar year association was observed in the long distance $\left(\chi^{2}=42.440, p<0.001\right.$, $\varphi=0.106)$ with the lowest MWR in 2014 (3.68) and highest in 2003 (12.95). No difference was shown in the short distance $\left(\chi^{2}=7.004, p=0.935, \varphi=0.041\right)$.
Regarding the total race time, men were faster than women by $8.2 \%$ (171 \pm 21 minutes vs. $186 \pm 21$ minutes, $p$ $\left.<0.001, \eta^{2}=0.068\right)$ and $7.5 \%(502 \pm 57$ minutes vs. 543 \pm 64 minutes, $\left.p<0.001, \eta^{2}=0.068\right)$ in the short and long distances, respectively. No change of race time across years was observed (Figure 2). The sex difference in race time increased in the long distance (e.g., from $4.9 \%$ in 2003 to $8.3 \%$ in $2017 ; p=0.014$ ), whereas it did not change in the short distance. No sex $\times$ calendar year interaction on race time was shown neither in the short distance $\left(p=0.465, \eta^{2}=0.003\right)$ nor in the long distance $\left(p=0.385, \eta^{2}=0.004\right)$.

Women were younger than men by 4.6 years $(35.0 \pm$ 9.0 years vs. $39.6 \pm 10.5$ years, $\left.p<0.001, \eta^{2}=0.026\right)$ and 1.8 years $(38.8 \pm 8.7$ years vs. $40.6 \pm 9.5$ years, $p<0.001$, $\left.\eta^{2}=0.005\right)$ in the short and long distances, respectively. Age increased across years in both sexes and distances $(p$ $<0.001$ ) (Figure 3). The sex difference in age decreased in the long distance $(p=0.007)$, but not in the short distance. Regarding the weather conditions, temperature, precipitation, and wind did not correlate with performance.
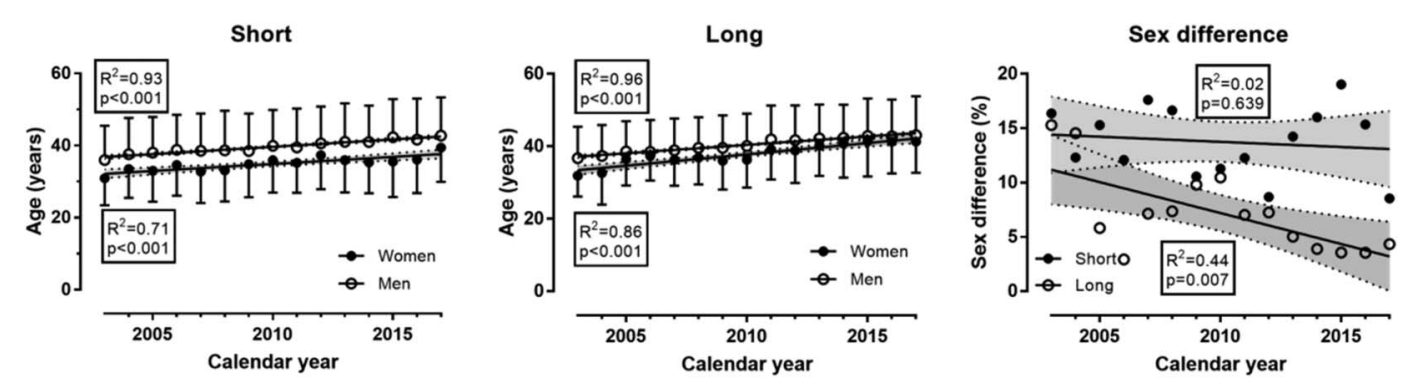

Figure 3. Age by calendar year and short vs. long distance in women and men. The dotted lines and gray areas represent $95 \%$ confidence intervals. Error bars show $S D$ s. $R^{2}=$ correlation of determination. Sex difference in age was calculated as "100 × (age in men - age in women)/age in women."

$4 \quad$ Journal of Strength and Conditioning Research 


\section{Discussion}

The main findings of this study were that (a) the participation of women increased and the MWR decreased in the long distance across years, (b) race times remained unchanged across years in both sexes and distances, (c) the sex difference in performance increased across years in the long distance, (d) women were younger than men, and (e) the age of women and men increased in both distances and the sex difference in age decreased in the long distance across years.

A first important finding was that the participation of women increased only in the long distance, whereas the number of men finishers remained stable in both distances across calendar years. An explanation of the discrepancy between the 2 distances might be the initial lower number of female finishers in the long distance, which allowed for a larger increase compared with the short-distance race. The initial lower rates of female finishers in the long-distance race were in agreement with research in triathlon showing a lower participation of women as the distance increased (18). Accordingly, the MWR decreased across calendar years only in the long-distance race, where it was initially higher than that in the short-distance race.

Competing in the longer race distance compared with the shorter race distance might also have motivational reasons. When ultra-marathoners competing in the same event in 12 and 24 hours were compared, the number of finishes was 3 times higher in the 24-hour run than the number of finishes in the 12-hour run (38). Indeed, the distance in an ultra-race seems not to be a limit in female participation. When the participation and performance trends in ultra-triathlons for Double Iron to Deca Iron ultra-triathlon between 1985 (i.e., first Double Iron ultra-triathlon in history) and 2009 were investigated, women accounted for both Double and Deca Iron ultra-triathlon $\sim 10 \%$ of the successful finishers (11). Although the abovementioned studies were conducted in ultra-endurance sports with longer duration than the 2 distances of duathlon examined in this study, they provided the opportunity to study participation in 2 different distances of ultra-endurance sport.

A second important finding was that performance in both women and men remained unchanged across years. This is in contrast to elite athletes competing in the Ironman triathlon World Championship, the "Ironman Hawaii." When the annual top 10 women and men competing in the race between 1983 and 2012 were analyzed, their race times decreased and therefore their performance improved (5). The most likely explanation that performance remained unchanged in the World Championship in duathlon compared with the improved performance in the World Championship in Ironman triathlon could be the time frame (e.g., shorter time frame in the World Championship in duathlon compared with the Ironman World Championship) and the different samples of athletes (i.e., annual top
10 women and men in the World Championship in Ironman triathlon compared with all women and men in World Championship in duathlon). However, also in the 12- and 24-hour run held in Basel, Switzerland, between 1988 and 2012, no changes in performance could be detected. The running speed for both the annual winners and the annual top 3 athletes showed no change across years neither in the 12-hour run nor in the 24-hour run (38).

A third important finding was that the sex difference in performance was similar in the 2 versions of duathlon (men were faster than women by $\sim 8 \%)$ and was lower than that reported in the Olympic triathlon (40).

Differences were found, however, for the trend in sex difference over time. In the long-distance race, the sex difference increased across years (i.e., women were not able to reduce the gap to men), whereas the sex difference showed no change in the short-distance race. The trend in sex difference in endurance and ultra-endurance over time seems to depend on the length of the performance, the kind of the discipline, and the MWR. Generally, women reduced the gap to men over time in long-distance swimming $(4,14,29)$, long-distance cycling (31), longdistance running $(15,24)$, and long-distance triathlon $(16,19,30)$.

The length of the race distance seems to be important for the trend in sex difference in performance. In long-distance triathlon races, women could reduce the sex difference only in shorter distances (e.g., Ironman triathlon) but not in longer triathlon distances (e.g., Double Iron ultra-triathlon and longer distances), where the sex difference remained unchanged or even increased over time, especially for the longer distances $(16,28,30,33)$.

In addition to the distance of the race (the longer the distance, the larger the sex difference in performance), another aspect related to sex difference is the relative participation of women and men expressed by the MWR. It has been shown previously in ultra-marathons that the sex difference in performance was larger when there were fewer women than men finishers in a race (32). Moreover, when all finishers competing in ultra-marathons from 6 hours to 10 days between 1975 and 2013 were analyzed, women reduced the gap to men for most of timed ultra-marathons and for those age groups where they had relatively high participation (15).

Considering that duathlon is an endurance sport, this sex difference in performance should be attributed to respective differences in physiological characteristics. It has been shown that duathletes possess high maximal oxygen uptake $\left(\dot{\mathrm{V}}_{2} \mathrm{max}\right)$, which, in turn, correlates with performance (39). In addition, aerobic capacity estimated by maximal workload during a graded exercise test was the best predictor of duathlon performance (3). Although no data on sex difference in duathletes' ${ }^{\mathrm{V}} \mathrm{O}_{2}$ max exist, it has been observed that men triathletes exhibit higher $\dot{\mathrm{V}}_{2}$ max than their women counterparts $(7,12)$. 
A fourth important finding was that women were younger than men in both race distances, and the sex difference in age was larger in the shorter race distance. This finding confirms recent findings for marathon runners. For instance, female marathoners competing in the "New York City Marathon" between 2006 and 2016 were $\sim 5$ years younger than male marathoners (23). In another study investigating marathoners competing in city marathons held in Switzerland, women were $\sim 3$ years younger than men (13).

When we consider duathlon as a multisports discipline like triathlon, we would expect that female long-distance triathletes would also achieve their best performance at a younger age compared with male long-distance triathletes. However, when the age of the top 10 finishers for all qualifier races, as well as for the Ironman World Championship event, itself was determined in 2010, women and men peaked at a similar age of $\sim 32-33$ years with no sex difference (35).

Most probably, the length of an endurance performance has also an effect on the sex difference in the age of peak performance. When $50-\mathrm{km}$ ultra-marathoners were analyzed, the age of peak running speed was 41 years in both women and men (21). When $100-\mathrm{km}$ ultra-marathoners were analyzed, women were faster than men. When female and male finishers were analyzed in 1-year age group intervals, the age of peak performance was at 41 years in women and 45 years in men when considering all finishers. When the top 10 finishers were considered, the age of peak performance was at 39 years in women and 41 years in men (22).

A fifth important finding was that age in both women and men increased over time. This confirms earlier findings for Ironman triathletes competing between 1983 and 2012 in the Ironman World Championship in "Ironman Hawaii." When the annual top 10 women and men were analyzed, their age increased over time (5). This could, however, not be confirmed when the age of the annual top 10 female and male finishers in a qualifier for "Ironman Hawaii" was determined in "Ironman Switzerland" between 1995 and 2011. During the study period, the age of peak performance remained unchanged for men at $31 \pm 3$ years, but increased only for women from $30 \pm 4$ years in 1995 to $36 \pm 5$ years in 2011 (27). Most probably, a change in age of elite endurance and ultra-endurance athletes depends on whether only top athletes (e.g., annual 10 fastest) or all athletes per calendar year are included in an analysis.

A limitation of this study was that since duathlon has not a standard format and the distances of first run, bike, and second run might vary (e.g., 5-20-2.5 km (25), 5-40-5 km (36), and $5-30-10 \mathrm{~km}(20)$ ), caution would be needed to generalize the findings of the "Powerman Zofingen" to other duathlons. In addition to the variation of distance among duathlons, the effect of environmental conditions should be considered. For instance, it has been shown that performance in duathlon was faster at $10^{\circ} \mathrm{C}$ than at $30^{\circ} \mathrm{C}$, suggesting that temperature might influence performance (34). For our analysis, we had access to 3 major environmental con- ditions (i.e., temperature, precipitation, and wind). The lack of correlation of these conditions with performance might be due to the limited available environmental data. For instance, daily maximum temperature, daily precipitation, and daily average wind speed were available; thus, we have no knowledge about the precise weather conditions during the race. Despite the lack of correlation between performance and weather, there was an indication about a likely relationship; for instance, compared to the previous and following calendar years, a relatively low performance occurred in 2006, when a high precipitation was observed. On the other hand, strength of this study was its novelty, as it was the first to examine participation and performance in different distances of a duathlon across years. Considering the increased popularity of duathlon, our findings have practical applications for coaches and fitness trainers working with duathletes in the context of long-term athlete's development. The knowledge of sex and age difference in performance is necessary to develop sex- and age-tailored training programs.

\section{Practical Applications}

The number of women finishers increased and the MWR decreased in the long distance. The increase of the sex difference in race time in the long distance might be attributed to the increased number of women finishers. On the contrary, the respective trends in the short distance remained stable. Thus, the trends in participation, performance, and age of finishers in "Powerman Zofingen" were distance-specific, and strength and conditioning coaches working with duathletes should consider these trends. For instance, the increasing popularity of this race might represent a general trend that duathlon becomes popular drawing attention to the need for professional support to duathletes from specialized exercise practitioners as well as for scientific research in this sport. Based on the findings of this study, strength and conditioning coaches should be aware of the increased participation of women in multisports, such as duathlon, especially in those sports where women had small participation previously, and of the fact that an increased participation is accompanied by a decreased performance. Accordingly, our results highlighted the need that strength and conditioning coaches working with duathletes and athletes of other multisports should be prepared to develop training programs tailored for slower and older athletes. It is important to mention that race times remained unchanged across years in both sexes and distances, although the age of women and men increased in both distances. In other words, the finishers become older but not slower-the pure descriptive feature of this study. As shown recently in 2 ultraendurance sports such as 50 - and $100-\mathrm{km}$ ultra-marathon running $(21,22)$, finishers became older and slower during the past decades, and our findings confirmed these trends of age and performance. In this context, strength and conditioning coaches should develop specific interest for "recreational" duathletes of older age.

6 Journal of Strength and Conditioning Research 


\section{REFERENCES}

1. Bolognesi, M. Ventilatory threshold and maximal oxygen uptake during cycling and running in duathletes. Med Sport 50: 209-216, 1997.

2. Calbet, JAL, Chavarren, J, and Dorado, C. Running economy and delayed onset muscle soreness. J Sports Med Phys Fitness 41: 18-26, 2001.

3. Chavarren Cabrero, J, Jimenez Ramirez, J, Dorado Garcia, C, Ballesteros Martinez-Elorza, JM, and Lopez Calbet, JA. Prediction of performance in duathlon competititon. Archivos de Medicina del Deporte 14: 17-24, 1997.

4. Eichenberger, E, Knechtle, B, Knechtle, P, Rust, CA, Rosemann, T, and Lepers, R. Best performances by men and women open-water swimmers during the "English Channel Swim" from 1900 to 2010. J Sports Sci 30: 1295-1301, 2012.

5. Gallmann, D, Knechtle, B, Rust, CA, Rosemann, T, and Lepers, R. Elite triathletes in "Ironman Hawaii" get older but faster. Age 36: 407-416, 2014

6. Garside, I and Doran, DA. Effects of bicycle frame ergonomics on triathlon 10-km running performance. J Sports Sci 18: 825-833, 2000.

7. González-Parra, G, Mora, R, and Hoeger, B. Maximal oxygen consumption in national elite triathletes that train in high altitude. J Hum Sport Exerc 8: 342-349, 2013

8. Granskog, J. Just "tri" and "du" it: The variable impact of female involvement in the triathlon/duathlon sport culture. In: Athletic Intruders: Ethnographic Research on Women, Culture, and Exercise. Albany, NY: State University of New York Press, 2003. pp. 27-52.

9. Hopkins, WG, Marshall, SW, Batterham, AM, and Hanin, J. Progressive statistics for studies in sports medicine and exercise science. Med Sci Sports Exerc 41: 3-13, 2009.

10. Iuliano, S, Naughton, G, Collier, G, and Carlson, J. Examination of the self-selected fluid intake practices by junior athletes during a simulated duathlon event. Int J Sport Nutr Exerc Metabol 8: 10-23, 1998

11. Knechtle, B, Knechtle, P, and Lepers, R. Participation and performance trends in ultra-triathlons from 1985 to 2009. Scand J Med Sci Sports 21: e82-90, 2011.

12. Knechtle, B, Muller, G, Willmann, F, Kotteck, K, Eser, P, and Knecht, H. Fat oxidation in men and women endurance athletes in running and cycling. Int J Sports Med 25: 38-44, 2004.

13. Knechtle, B, Nikolaidis, PT, Zingg, MA, Rosemann, T, and Rust, CA. Differences in age of peak marathon performance between mountain and city marathon running-The "Jungfrau Marathon" in Switzerland. Chin J Physiol 60: 11-22, 2017.

14. Knechtle, B, Rosemann, T, and Rust, CA. Women cross the “Catalina Channel” faster than men. Springerplus 4: 332, 2015.

15. Knechtle, B, Valeri, F, Nikolaidis, PT, Zingg, MA, Rosemann, T, and Rust, CA. Do women reduce the gap to men in ultra-marathon running? Springerplus 5: 672, 2016.

16. Knechtle, B, Zingg, MA, Rosemann, T, and Rust, CA. Sex difference in top performers from Ironman to Double Deca Iron ultratriathlon. Open Access J Sports Med 5: 159-172, 2014.

17. Lane, A, Terry, $\mathrm{P}$, and Karageorghis, C. Antecedents of multidimensional competitive state anxiety and self-confidence in duathletes. Percept Mot Skills 80: 911-919, 1995.

18. Lepers, R, Knechtle, B, and Stapley, PJ. Trends in triathlon performance: Effects of sex and age. Sports Med 43: 851-863, 2013.

19. Meili, D, Knechtle, B, Rust, CA, Rosemann, T, and Lepers, R. Participation and performance trends in "Ultraman Hawaii" from 1983 to 2012. Extrem Physiol Med 2: 25, 2013.

20. Moncada-Jiménez, J, Plaisance, E, Mestek, ML, Araya-Ramírez, F, Ratcliff, L, Taylor, JK, et al. Initial metabolic state and exerciseinduced endotoxaemia are unrelated to gastrointestinal symptoms during exercise. J Sports Sci Med 8: 252-258, 2009.
21. Nikolaidis, PT and Knechtle, B. Age of peak performance in 50-km ultramarathoners-Is it older than in marathoners? Open Access $J$ Sports Med 9: 37-45, 2018.

22. Nikolaidis, PT and Knechtle, B. Performance in 100-km ultramarathoners-At which age it reaches its peak? J Strength Cond Res, 2018. Epub ahead of print.

23. Nikolaidis, PT, Rosemann, T, and Knechtle, B. Sex differences in the age of peak marathon race time. Chin J Physiol 61: 85-91, 2018.

24. Peter, L, Rust, CA, Knechtle, B, Rosemann, T, and Lepers, R. Sex differences in 24-hour ultra-marathon performance-a retrospective data analysis from 1977 to 2012. Clinics 69: 38-46, 2014.

25. Ronconi, M and Alvero-Cruz, JR. Heart rate and oxygen uptake responses in male athletes in duathlon sprint competitions. Apunts Medicina de l'Esport 46: 183-188, 2011.

26. Rust, CA, Knechtle, B, Knechtle, P, Pfeifer, S, Rosemann, T, Lepers, $\mathrm{R}$, et al. Gender difference and age-related changes in performance at the long-distance duathlon. J Strength Cond Res 27: 293-301, 2013.

27. Rust, CA, Knechtle, B, Knechtle, P, Rosemann, T, and Lepers, R. Age of peak performance in elite male and female Ironman triathletes competing in Ironman Switzerland, a qualifier for the Ironman world championship, Ironman Hawaii, from 1995 to 2011. Open Access J Sports Med 3: 175-182, 2012.

28. Rust, CA, Knechtle, B, Knechtle, P, Rosemann, T, and Lepers, R. Sex differences in ultra-triathlon performance at increasing race distance. Percept Mot Skills 116: 690-706, 2013.

29. Rust, CA, Lepers, R, Rosemann, T, and Knechtle, B. Will women soon outperform men in open-water ultra-distance swimming in the "Maratona del Golfo Capri-Napoli”? Springerplus 3: 86, 2014.

30. Rust, CA, Rosemann, T, and Knechtle, B. Performance and sex difference in ultra-triathlon performance from Ironman to Double Deca Iron ultra-triathlon between 1978 and 2013. Springerplus 3: 219,2014

31. Rust, CA, Rosemann, T, Lepers, R, and Knechtle, B. Gender difference in cycling speed and age of winning performers in ultracycling - the 508-mile "Furnace Creek" from 1983 to 2012. J Sports Sci 33: 198-210, 2015.

32. Senefeld, J, Smith, C, and Hunter, SK. Sex differences in participation, performance, and age of ultramarathon runners. Int $J$ Sports Physiol Perform 11: 635-642, 2016.

33. Sigg, K, Knechtle, B, Rust, CA, Knechtle, P, Lepers, R, and Rosemann, T. Sex difference in Double Iron ultra-triathlon performance. Extrem Physiol Med 2: 12, 2013.

34. Sparks, SA, Cable, NT, Doran, DA, and Maclaren, DPM. The influence of environmental temperature on duathlon performance. Ergon 48: 1558-1567, 2005.

35. Stiefel, M, Knechtle, B, Rust, CA, Rosemann, T, and Lepers, R. The age of peak performance in Ironman triathlon: A cross-sectional and longitudinal data analysis. Extrem Physiol Med 2: 27, 2013.

36. Suzuki, K, Shiraishi, K, Yoshitani, K, Sugama, K, and Kometani, T. Effect of a sports drink based on highly-branched cyclic dextrin on cytokine responses to exhaustive endurance exercise. J Sports Med Phys Fitness 54: 622-630, 2014.

37. Tauler, P, Aguiló, A, Gimeno, I, Fuentespina, E, Tur, JA, and Pons, A. Influence of vitamin $\mathrm{C}$ diet supplementation on endogenous antioxidant defences during exhaustive exercise. Pflugers Arch Eur J Physiol 446: 658-664, 2003.

38. Teutsch, A, Rüst, CA, Knechtle, B, Knechtle, P, Rosemann, T, and Lepers, R. Differences in age and performance in 12-hour and 24hour ultra-runners. Adapt Med 5: 138-146, 2013.

39. Tong, RJ and Rees, JA. Determinants of performance in the duathlon. J Sports Sci 14: 58-59, 1996.

40. Wonerow, M, Rüst, CA, Nikolaidis, PT, Rosemann, T, and Knechtle, B. Performance trends in age group triathletes in the Olympic distance triathlon at the world championships 2009-2014. Chin J Physiol 60: 137-150, 2017. 